\title{
Harnessing antitumor immunity: Employment of tumor recall antigens to optimize the inflammatory response to cancer (Review)
}

\author{
KIMBERLY A. SILVERIO ${ }^{1}$ and SHYAM A. PATEL ${ }^{2}$ \\ ${ }^{1}$ Department of Gynecologic Oncology, Helen Diller Family Comprehensive Cancer Center, University of California, \\ San Francisco (UCSF), San Francisco, CA 94158; \\ ${ }^{2}$ Stanford Cancer Institute, Stanford University Medical Center, Stanford, CA 94305, USA
}

Received July 29, 2016; Accepted November 17, 2016

DOI: $10.3892 / \mathrm{ol} .2017 .5721$

\begin{abstract}
The advent of immunotherapy for cancer has contributed to the era of personalized medicine for cancer. The various immunotherapy-based treatments that have been explored thus far include monoclonal antibody therapy, tumor vaccines, immune checkpoint blockade and adoptive $\mathrm{T}$ cell transfer, among others. The groundwork for all these immunotherapeutic modalities rests within the tumor microenvironment, specifically the immune factors that influence the tumor-drug interface. Prior to therapeutic design, the tumor microenvironmental interactions and the current barriers to successful treatment must first be understood. In the present review, it is proposed that cancer cell eradication within the tumor niche may be achieved by reprogramming of the immune microenvironment in favor of a pro-inflammatory antitumor profile at an early stage. This pro-inflammatory profile may, in turn, be influenced by tumor recall antigens, which function to stimulate the cell-mediated or humoral responses involved in antitumor immunity. These measures serve to counteract the immunotolerant state of the tumor microenvironment. Such measures are critical to therapeutic successes.
\end{abstract}

\section{Contents}

1. Introduction

2. The immune microenvironment in the tumor niche

3. Regulatory $\mathrm{T}$ cells $\left(\mathrm{T}_{\mathrm{regs}}\right)$ in the microenvironment

4. Myeloid-derived suppressor cells (MDSCs) in the microenvironment

5. Temporal effect of inflammation in cancer

Correspondence to: Dr Shyam A. Patel, Stanford Cancer Institute, Stanford University Medical Center, 875 Blake Wilbur Drive, Stanford, CA 94305, USA

E-mail: shyam2@stanford.edu

Key words: regulatory $\mathrm{T}$ cells, immunotolerance, tumor recall antigens, cancer vaccine, microenvironment
6. Implications for immune checkpoint blockade in the microenvironment

7. Employment of tumor recall antigens

8. Future prospects on targeted immunotherapy

\section{Introduction}

For the past few decades, cytotoxic chemotherapy involving anti-metabolites has been the mainstay of the treatment of cancer (1). This type of therapy has improved overall survival and progression-free survival for thousands of patients with various types of cancer (1). However, in the majority of cases, it has also created a selective pressure that favored the accumulation of mutant cells with a survival advantage (2). Therefore, subsequent cytotoxic chemotherapy has become somewhat ineffective due to resistance (2). One of the most effective modalities of cancer treatment in recent years has been immunotherapy, principally monoclonal antibodies, vaccine-based therapies, immune checkpoint blockade and ex vivo therapy involving $\mathrm{T}$ cell priming with tumor antigens (3). Monoclonal antibody therapy has been used to deplete populations of cancer cells with specific phenotypes via antibody-dependent cell-mediated cytotoxicity or direct cytotoxicity (3). Vaccine-based therapy aims to prevent cancer development (prophylactic vaccination) or to eliminate cancer cells (therapeutic vaccination) (4). The advent of immune checkpoint blockade, specifically programmed cell death protein 1 (PD-1) /PD-ligand 1 (L1) and cytotoxic T-lymphocyte (CTL)-associated protein 4 (CTLA-4) inhibition, is a prime example of how tumor immunotherapy has been translated into practice with significant success $(5,6)$. Current therapeutic strategies in clinical oncology are taking advantage of the natural killer cell (NK)-mediated and CTL-mediated anticancer responses $(3,7)$. The mechanisms underlying the aforementioned therapies are founded upon immunological crosstalk in the tumor microenvironment at an early time point, prior to the development of advanced-stage cancer $(3,7)$. The current review discusses the requirement for strategies to reprogram the immune microenvironment of cancer cells in favor of a pro-inflammatory anticancer response at an early stage. This is particularly important given the variety of cell types in the microenvironment that influence tumor behavior (8). The specified goals may be achieved by the use of tumor recall antigens. 


\section{The immune microenvironment in the tumor niche}

In order to appreciate the significance of immunotherapy, the tumor microenvironment must be understood. Paget et al (9) first proposed the concept of the tumor microenvironment over 120 years ago $(9,10)$. Paget et al (9) detailed the 'seed and soil' hypothesis for cancer, suggesting that a cancer cell ('seed') requires a conductive niche ('soil') in order to survive. The tumor microenvironment is composed of a variety of cells, including epithelial cells, stromal cells, fibroblasts and immune cells (8). Non-cellular components of the microenvironment include cytokines, chemokines, growth factors and other soluble mediators (8). Paget's framework gained significant support $(9,10)$. Recently, novel targeted therapies have been developed in a manner that allows for elimination of cancer cells by altering neighboring cellular components of the microenvironment (10). A prime example of this is the inhibition of angiogenesis by monoclonal antibodies: Microenvironmental targeting agents, including anti-vascular endothelial growth factor (VEGF) therapies, result in the halting of angiogenic processes that normally provide nourishment for tumor cells (10).

Perhaps the most important amongst the cells of the tumor microenvironment are the immune cells (8). Immune cells in the tumor niche include CTLs, helper T (Th) lymphocytes, $\mathrm{T}_{\text {regs }}$, MDSCs and tumor-associated macrophages (TAMs), among others (8) (Table I). The products of these various cells include pro-inflammatory cytokines, specifically interleukin (IL)-6, IL-17, IL-22 and tumor necrosis factor (TNF)- $\alpha$ (8). Although cytokines are able to function in antitumor immunity, they may also serve to promote cellular proliferation (8). For example, IL-6 is a pro-inflammatory cytokine that has pleotropic effects, and a large body of evidence has revealed that it sustains the pro-tumor milieu (11). However, it is also involved in the inhibition of $\mathrm{T}_{\text {reg }}$ function and allows effector $\mathrm{T}$ cells to avoid suppression by $\mathrm{T}_{\text {regs }}$, which favors tumor cell elimination (11). Conversely, transforming growth factor (TGF) $-\beta$ production by the immune microenvironment may polarize naïve $\mathrm{T}$ cells towards the $\mathrm{T}_{\mathrm{reg}}$ lineage, favoring the immune escape of tumor cells (7). The effects of various immune cells are, therefore, bimodal. Immune subsets, including $\mathrm{T}_{\text {regs }}$ and MDSCs, co-exist with tumor cells to create an immunotolerant state (7). These key cellular factors of the immune microenvironment and their relevance to cancer are discussed in the present review.

\section{Regulatory $T$ cells $\left(T_{\text {regs }}\right)$ in the microenvironment}

$\mathrm{T}_{\text {regs }}$ comprise a small population of lymphocytes initially detailed in the 1970s; they were identified to be thymocytes able induce tolerance in murine models (12). $\mathrm{T}_{\text {regs }}$ function in the prevention of autoimmunity, the maintenance of immune homeostasis and the maintenance of self-tolerance (13). By the $1980 \mathrm{~s}$, it was noted that a suppressor T cell population was involved in the immune escape of cancer cells (13). From that time, the role of $T_{\text {regs }}$ in cancer has been further elucidated and, phenotypically, $\mathrm{T}_{\mathrm{regs}}$ are characterized as $\mathrm{CD} 4{ }^{+} \mathrm{CD} 25^{+}$forkhead box protein $3(\mathrm{FOXP} 3)^{+}(13)$ Functionally, $\mathrm{T}_{\text {regs }}$ secrete IL-10, TGF- $\beta$ and various other cytokines that exert downstream pan-immunosuppressive effects (13). $T_{\text {regs }}$ within the microenvironment have a central role in tumor maintenance via the suppression of antitumor immune responses (7). Anti-inflammatory cytokines from $\mathrm{T}_{\text {regs }}$ may serve to prevent pro-inflammatory responses that normally destroy cancer cells (7). In breast cancer, for example, it has previously been demonstrated that $\mathrm{T}_{\text {regs }}$ inhibit $\mathrm{T}$ cell proliferation and migration, as well as NK cell and CTL function, ultimately contributing to breast cancer cell survival (7). These actions are mediated by TGF- $\beta$ from bone marrow-derived mesenchymal stem cells, which induce $\mathrm{T}_{\text {regs }}$ (7). Subsequently, TGF- $\beta$ has been revealed to have a role in other types of cancer; TGF- $\beta$ is associated with the increased aggressiveness of prostate cancer (14). Infiltration of $\mathrm{T}_{\text {regs }}$ within tumor tissues carries an overall worse prognosis (15).

Strategies for optimization of the inflammatory response within the tumor microenvironment include the modulation of $\mathrm{T}_{\text {regg }}$; this may occur via $\mathrm{T}_{\text {reg }}$ depletion or through the modulation of $\mathrm{T}_{\text {reg }}$ trafficking (16). Depletion may be achieved with anti-cluster of differentiation (CD) 25 antibodies, and $\mathrm{T}_{\text {reg }}$ trafficking may be manipulated by targeting chemokine/receptor pairs that govern the migration of $T_{\text {regs }}$ into the tumor microenvironment (16). The eventual therapeutic goal is to minimize $T_{\text {reg }}$ frequency and/or to hinder $T_{\text {reg }}$ function within the tumor niche, as this will prevent the development of an immune tolerance state and, therefore, prevent cancer cell protection.

\section{Myeloid-derived suppressor cells (MDSCs) in the micro- environment}

MDSCs comprise a heterogeneous population of immune cells of myeloid origin (17). Similarly to $T_{\text {regs }}$, MDSCs represent only a small fraction of all immune cells from peripheral blood (17). These cells are phenotypically characterized as

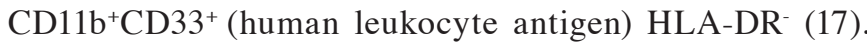
They are functionally characterized by their potent immunosuppressive ability, which, as with $\mathrm{T}_{\text {regs }}$, is able to inhibit antitumor immune responses (17). Mechanisms underlying immunosuppression effected by MDSCs comprise, but are not limited to, the consumption of essential amino acids, including arginine, which is used as a substrate for inducible nitric oxide synthase, resulting in the generation of nitric oxide (18). Nitric oxide is a reactive oxygen species that impairs antigen-presenting cell (APC) function and causes the apoptosis of tumor-specific CTLs (18).

Thus far, the role of MDSCs has extended to multiple types of cancer. Previous studies have demonstrated that MDSCs are able to inhibit $\mathrm{T}$ cell proliferation in extranodal lymphoma (19). There is an increased number of MDSCs in patients with lymphoma compared with healthy patients, and a high MDSC fraction is associated with a worse prognosis (17). These clinical correlations are similar to those observed for $\mathrm{T}_{\text {regs }}$; immunosuppressive subsets within the tumor stroma are associated with worse patient treatment outcomes (17). The presence of MDSCs in the tumor microenvironment may induce a state of immunotolerance, whereby tumor cells are resistant to immune-mediated destruction (17). The migration of MDSCs in cancer has also been explored. A recent study in gastric cancer has indicated that MDSC trafficking involves 
Table I. Key cellular factors in the tumor microenvironment.

\begin{tabular}{|c|c|c|c|}
\hline Cell Type & Phenotype & Function & Reference \\
\hline Epithelial cells & Tissue-specific & General cellular support & $(8)$ \\
\hline Stromal cells & $\mathrm{CD} 44^{+} \mathrm{CD} 90^{+}$ & Structural support, cytokine provision for tumor growth & (8) \\
\hline Endothelial cells & $\mathrm{CD} 31^{+} \mathrm{VCAM}^{-1}{ }^{+}$ & Vascular support & $(8)$ \\
\hline MDSCs & 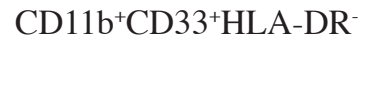 & $\begin{array}{l}\text { Inhibition of antitumor immune responses; } \\
\text { triggering apoptosis of CTLs }\end{array}$ & $(17-18)$ \\
\hline $\mathrm{T}_{\text {regs }}$ & $\mathrm{CD} 4^{+} \mathrm{CD} 25^{+} \mathrm{FoxP} 3^{+}$ & $\begin{array}{l}\text { Maintenance of immune homeostasis, inhibition of } \\
\text { anti-tumor immune responses }\end{array}$ & (13) \\
\hline M1 TAMs & $\mathrm{CD}^{6} 8^{+} \mathrm{CD} 86^{+} \mathrm{TNF}-\alpha^{+}$ & Activation of the antitumor effect; immune stimulation & $(8)$ \\
\hline M2 TAMs & $\mathrm{CD} 68^{+} \mathrm{CD} 206^{+} \mathrm{CD} 163^{+}$ & Suppression of the antitumor effect; enhancement of tumor growth & (8) \\
\hline
\end{tabular}

the $\mathrm{C}-\mathrm{X}-\mathrm{C}$ motif chemokine receptor (CXCR) 5/C-X-C motif ligand 13 signaling pathway within the tumor microenvironment (20). The CXCR5-dependent migration of MDSCs towards tumor cells is, therefore, a potentially targetable interaction in the tumor microenvironment.

\section{Temporal effect of inflammation in cancer}

It has previously been recognized that inflammation serves a dual role in neoplasia: A pro-inflammatory response may hinder and also promote cancer cell growth and metastasis (21). In the presence of inflammation induced by stress, injury or systemic disease, inflammatory mediators act directly on cancer cells, or indirectly on other factors of the tumor microenvironment, in order to promote cancer progression (21). A recent study demonstrated that the activation of $\mathrm{C}-\mathrm{C}$ motif chemokine ligand-2/C-C chemokine receptor type-2 signal transduction in macrophages may alter their phenotype such that the macrophages promote breast cancer cell metastasis (21). These types of studies emphasize the increasingly important role of chemokine antagonists in cancer therapy.

The temporal effect of inflammation may also be bimodal; the early and acute inflammatory response may result in antitumor immunity, but a delayed and chronic inflammatory response may contribute to tumor maintenance or progression $(4,22)$. There is currently no established data supporting the time-dependent bimodal effects of inflammation, but it is proposed in the present review that such a model may be representative of the microenvironment $(4,22)$. The early inflammatory response involving Th1 cells results in the production of various antitumor cytokines, which assist in the elimination of malignant cells (7). Tumor-infiltrating lymphocytes have previously been associated with improved pathological responses to cancer treatment. Th17-mediated CTL activation and production of interferon (IFN) $-\gamma$ assist in the antitumor immune response. Harnessing the immune system at this early stage is critical for eliminating cancer cells. However, once this acute inflammation settles, a more chronic inflammatory process characterized by Th2 cells and associated cytokines, in addition to TGF- $\beta$, is established (7). Chronic inflammation has been demonstrated to facilitate cancer progression via the generation of an immunosuppressive microenvironment (23). This often results in the activation of immune checkpoint pathways in lymphocytes (8). This chronic inflammatory response establishes a state of immune tolerance, allowing for the persistence or even the propagation of cancer cells within the microenvironment (8). Th17-mediated production of IL-17 in the tumor microenvironment facilitates the local expansion of the cancer cell population (4). The latter response serves to hinder antitumor immune responses in the microenvironment. Notably, a number of the same immune cell types, including Th17 cells, are involved in the acute and chronic inflammatory responses, but their secretomes and functions differ (4).

In addition to the Th17 polarization effect, macrophage polarization serves an important role in the tumor microenvironment. Classically activated macrophages (M1 macrophages) are induced by Th1-type cytokines, favoring antitumor immunity (4). Alternatively activated macrophages (M2 macrophages) are induced by Th2-cytokines, favoring immune protection and tumor growth (4).

If chronic inflammation does promote cancer progression, the question that arises is: Can the immune response within the tumor microenvironment be reprogrammed such that antitumor effects supersede the pro-tumor effects? Delineation of the temporal effect in the inflammatory niche requires additional investigation. Furthermore, investigation into a strategy that optimizes the frequency of intratumoral T lymphocytes and the Th1 response, without progression to an immunotolerant chronic inflammatory response, is essential to the elimination of cancer cells in the microenvironment. The answer to the aforementioned question lies in the optimization of contact-dependent interactions (tumor-stroma and tumor-immune interactions) and soluble mediators (chemokines) within the tumor microenvironment. 


\section{Implications for immune checkpoint blockade in the mi- croenvironment}

Immune checkpoint blockade has recently emerged as a therapy for multiple cancer types. Two cell-surface molecules on T lymphocytes that have a key role in the immune checkpoint are PD-1 and CTLA-4 (24). These are highly relevant immune checkpoint proteins that have exhibited clinical significance: Therapies have been developed to inhibit these checkpoints and, thus, promote the destruction of tumor cells (24). Nivolumab is an entirely human Immunoglobulin G4 against PD-1 that is Food and Drug Administration (FDA)-approved for the treatment of melanoma, advanced renal cell carcinoma following anti-VEGF therapy, metastatic non-small cell lung cancer following platinum-based therapy and of relapsed Hodgkin's lymphoma (25-27). Pembrolizumab is a PD-1 inhibitor that is FDA-approved for the treatment of metastatic melanoma, metastatic non-small cell lung cancer with PD-L1 expression and of head and neck cancer that is metastatic or recurrent following platinum-based therapy (28). Ipilimumab is a CTLA-4 inhibitor that is FDA-approved for the treatment of malignant melanoma (24). In addition to single-agent therapy, combination therapy with checkpoint inhibitors has demonstrated clinical effectiveness (24). In recent years, PD-1 and CTLA-4 targeting antibodies, individually and in combination, have exhibited significant clinical benefit in advanced melanoma, and the combination is now FDA-approved (24).

As immune checkpoint blockade continues to be pursued for the treatment of other types of cancer, including solid and hematological malignancies, it is essential to consider the potential associated uncontrolled immunological activation, which may contribute to a chronic inflammatory microenvironment leading to cancer progression $(4,22)$. Specifically, immunological activation and autoimmunity are complications, as checkpoint inhibitors disturb normal homeostasis (29). Common organ-specific adverse effects include nephritis, colitis and pneumonitis (30).

\section{Employment of tumor recall antigens}

In patients with cancer, the immune system is frequently the primary barrier that circulating cancer cells must overcome. These events occur during subclinical periods, often prior to the detection or diagnosis of cancer. Upon immune recognition of cancer cells, tumor-associated antigens are first presented to APCs (31). APCs then migrate to lymphoid beds that drain tumor cells and present the tumor-associated antigens via major histocompatibility complex (MHC) class II complexes to Th cells. Th cells, specifically Th1, release various cytokines, including IL-2, IFN- $\gamma$, and TNF- $\alpha$, which activate NK cells and CTLs; these cells ultimately serve as the effector cells in the immune response. Ex vivo priming of naïve T cells with tumor cells may generate tumor-specific T cells, and further amplification of the response may be achieved with the use of co-stimulatory molecules, including OX-40 (Fig. 1).

A recall antigen is an immunogenic molecule that is re-introduced into a person in whom initial exposure to the antigen resulted in sensitization. It is an antigen characterized by the ability to sensitize a person's immune system and to generate a powerful and rapid response upon repeat

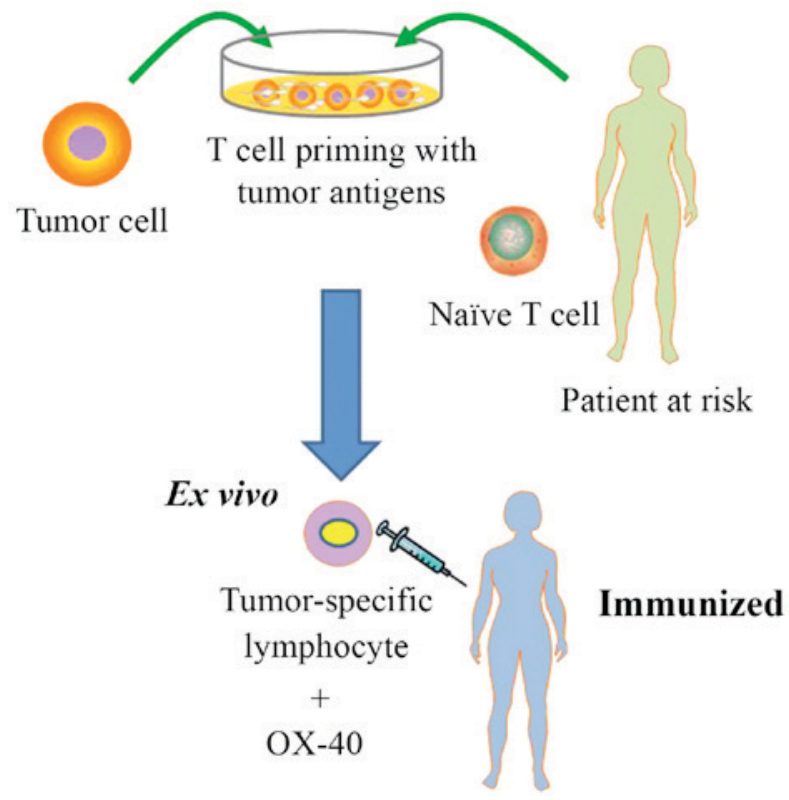

Figure 1. Immune-based ex vivo cancer therapy. Harvesting naïve T cells with tumor cells may lead to effective $T$ cell priming. These tumor-specific lymphocytes may be adoptively transferred into patients who are at risk for developing cancer. This is a form of secondary cancer prevention, whereby the development of cancer is impeded by a particular intervention in predisposed persons. Co-stimulatory molecules, including OX-40 may be employed to lead to more robust immune responses.

exposure (31). Tumor recall antigens may be employed with the aim of generating robust immune responses at an early stage (Fig. 2). The use of tumor recall antigens as a vaccination strategy must be evaluated with large-scale epidemiologic data in order to identify patients who may be at risk for developing cancer following a number of years, and to identify patients or populations who would benefit from the use of these antigens. Recall antigens are particularly appealing due to their immunogenic potential. In theory, the majority of tumor recall antigens are peptides, but they may also be carbohydrates, glycoproteins, lipids or other molecules capable of inducing an immune response (31). The recall antigens may be cell-surface molecules or intracytoplasmic molecules (31). Essentially, these antigens must be expressed in tumor cells but not in corresponding nonmalignant cells of the same tissue type (31). The employment of tumor recall antigens in conjunction with optimization of the immune microenvironment offers significant therapeutic potential for various types of cancer.

One example of a tumor recall antigen is citrullinated vimentin, which is presented on MHC class II molecules for recognition by $\mathrm{CD} 4^{+} \mathrm{Th}$ cells (32). Vimentin becomes a tumor marker during the process of the epithelial-mesenchymal transition, whereby cells acquire an invasive phenotype that promotes metastasis (32). The process of tumor cell autophagy results in the release and exposure of tumor antigens to the naïve immune system (32). Other examples are cancer-testis antigens, including TEX19, which are expressed in tumors but not in normal tissues aside from the testes (33). Cancer-testis antigens are highly immunogenic; therefore, re-introduction may lead to a robust secondary response (33). Re-introduction of these antigens may serve as a therapeutic avenue for cancer. 

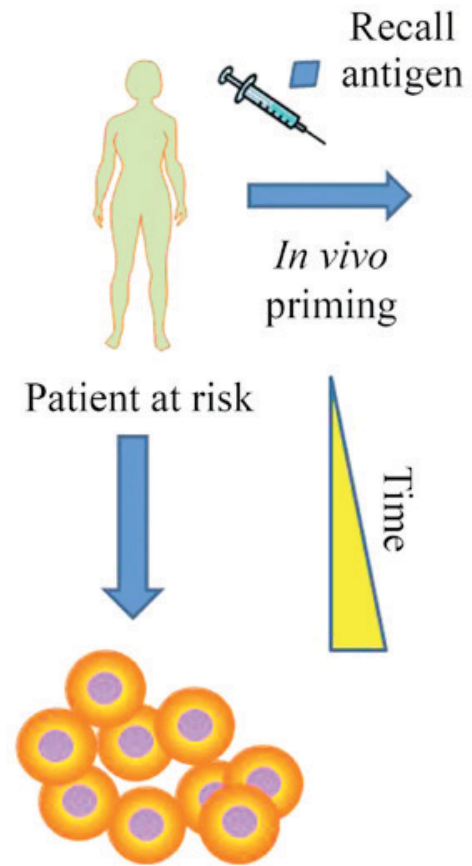

Cancer progression

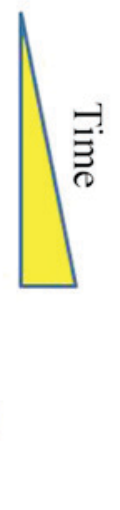

\section{No progression}

Figure 2. In vivo $\mathrm{T}$ cell priming with tumor recall antigens. Tumor recall antigens are immunogenic molecules that stimulate immunity on initial exposure. In patients who are not immunized, subclinical tumorigenesis may progress to a candid diagnosis of cancer. During periods of subclinical tumorigenesis, when circulating tumor cells are present but cancer is clinically detectable, tumor recall antigens are able to generate a robust anamnestic response. This is particularly important in the early stages of tumor immunity. Tumor recall antigens may be utilized to generate durable immune-based treatment responses.

\section{Future prospects on targeted immunotherapy}

As the potential of targeted cancer therapy and personalized medicine improves, the following question must be presented: What resistance mechanisms will cancer cells acquire in response to the selective pressure of novel agents? The acquisition of mutations in cancer cells and the clonal evolution of cells towards resistant phenotypes appear to be inevitable and the anticipation of these events will be critical. Traditionally, the approach has been to respond to these changes in a retrospective manner, a strategy that results in an immunotolerant state leading to the survival of cancer cells (Fig. 3A). However, the most beneficial approach is a prospective one, in which cancer cells may be eliminated by targeting their inherent properties prior to the development of mutations and resistance (Fig. 3B). This may potentially be achieved by optimizing the immune microenvironment and tumor niche (7). Reprogramming of the immune microenvironment in a manner that disturbs the immunotolerant state will be critical for therapeutic successes.

Microenvironmental barriers to the success of immunotherapy include the presence of intratumoral $\mathrm{T}_{\text {regs }}$ and MDSCs. These immunosuppressive cell populations are able to inhibit the normal local antitumor response. Thus, optimization of the intratumoral microenvironment by polarizing immune cells away from immune-mediated cancer protection is crucial to the success of immunotherapy (7). It is proposed in the current review that a local mode switch from an immunotolerant phase
$\mathbf{A}$
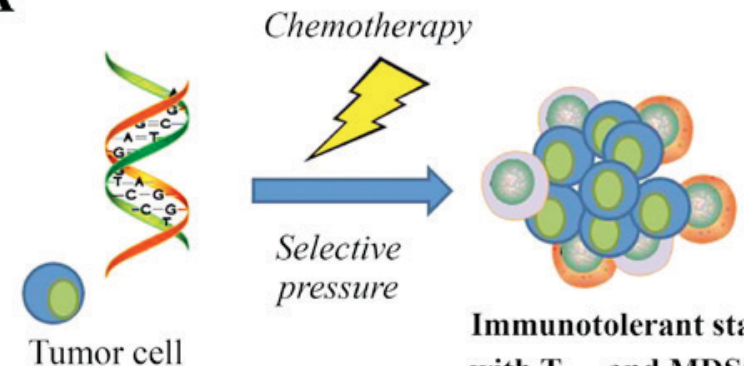

Immunotolerant state with $\mathrm{T}_{\text {regs }}$ and MDSCs

B
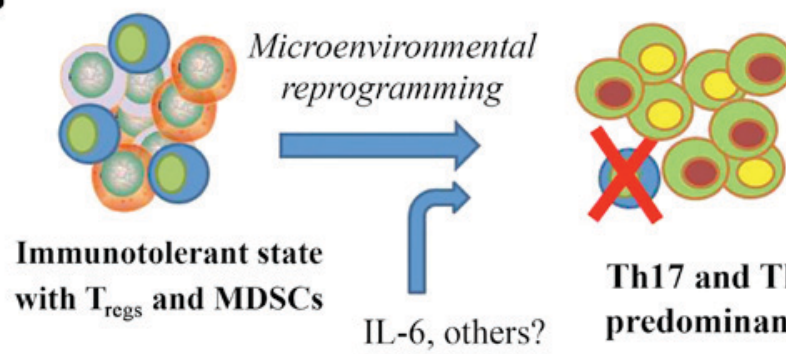

Th17 and Th1 predominance

Figure 3. Strategies for microenvironmental reprogramming. (A) Selective pressure is inevitable when cancer cells are initially treated with chemotherapy and a fraction of resistant cells survive. Re-treatment with another agent at a later time results in the propagation of mutants. Eventually, the immune microenvironment establishes an immunotolerant state, with $\mathrm{T}_{\text {reg }}$ and MDSC predominance. Co-adaptation of tumor cells and these immunosuppressive subsets leads to the persistence of cancer. (B) Reprogramming of the tumor microenvironment involves transition from an immunotolerant state to an immunostimulatory state. Immunostimulatory subsets include Th1 and Th17 cells, which promote antitumor immunity. One such molecular switch involved in reprogramming is IL-6. Other molecular switches remain to be explored. $\mathrm{T}_{\text {reg }}$, regulatory $\mathrm{T}$ cells; MDSC, myeloid-derived suppressor cells; Th, helper T lymphocytes; IL, interleukin.

to an immunostimulatory phase, or from a chronic inflammatory phase to an acute inflammatory phase may assist the elimination of cancer. A specific molecular mediator that must be exploited is IL-6, as this cytokine is involved in the early inflammatory milieu and the suppression of $\mathrm{T}_{\text {reg }}$ function (34). IL-6 functions in the activation of the Th17 transcription factor RAR-related orphan receptor (ROR)- $\gamma$ t and in the inhibition of the $\mathrm{T}_{\text {reg }}$ transcription factor FOXP3 (34). Other molecular mediators that govern the switch from chronic to acute inflammation must also be explored.

Future treatment strategies must focus on re-directing immunosuppressive networks within tumor cell microenvironments. The recent FDA approval of the first oncolytic viral therapy, talimogene laherparepvec, in October 2015 has highlighted the potential for novel modalities of cancer therapy (35). These therapeutic viruses are important factors in the immune microenvironment as they are able to induce an immune response to themselves as well as their target cancer cells (36). Oncolytic viruses may serve as delivery vehicles of anticancer genes or of genes that promote antitumor immune responses, including granulocyte-macrophage colony-stimulating factor (36). Delineation of the immunological changes that are mediated by oncolytic viral therapy for various type of cancer deserves further merit. The use of a tumor-infecting virus as the delivery vehicle of an immunostimulatory agent has paved the way for future modalities of cancer therapy. 
These aspects of tumor immunology are becoming critically important in the era of targeted therapy.

\section{References}

1. Milsted RA, Tattersall MH, Fox RM and Woods RL: Cancer chemotherapy-what have we achieved? Lancet 1: 1343-1346, 1980.

2. Welch AD: The problem of drug resistance in cancer chemotherapy. Cancer Res 19: 359-371, 1959.

3. Press OW, Appelbaum F, Ledbetter JA, Martin PJ, Zarling J, Kidd P and Thomas ED: Monoclonal antibody 1F5 (anti-CD20) serotherapy of human B cell lymphomas. Blood 69: 584-591, 1987.

4. Roeser JC, Leach SD and McAllister F: Emerging strategies for cancer immunoprevention. Oncogene 34: 6029-6039, 2015.

5. Benson DM Jr, Bakan CE, Mishra A, Hofmeister CC, Efebera Y, Becknell B, Baiocchi RA, Zhang J, Yu J, Smith MK, et al: The PD-1/PD-L1 axis modulates the natural killer cell versus multiple myeloma effect: A therapeutic target for CT-011, a novel monoclonal anti-PD-1 antibody. Blood 116: 2286-2294, 2010.

6. Kwon ED, Hurwitz AA, Foster BA, Madias C, Feldhaus AL, Greenberg NM, Burg MB and Allison JP: Manipulation of T cell costimulatory and inhibitory signals for immunotherapy of prostate cancer. Proc Natl Acad Sci USA 94: 8099-8103, 1997.

7. Patel SA, Meyer JR, Greco SJ, Corcoran KE, Bryan M and Rameshwar P: Mesenchymal stem cells protect breast cancer cells through regulatory T cells: Role of mesenchymal stem cell-derived TGF-beta. J Immunol 184: 5885-5894, 2010.

8. Zamarron BF and Chen W: Dual roles of immune cells and their factors in cancer development and progression. Int J Biol Sci 7: 651-658, 2011.

9. Paget S: The distribution of secondary growths in cancer of the breast. 1889. Cancer Metastasis Rev 8: 98-101, 1889.

10. Langley RR and Fidler IJ: The seed and soil hypothesis revisited-the role of tumor-stroma interactions in metastasis to different organs. Int J Cancer 128: 2527-2535, 2011.

11. Goodman WA, Levine AD, Massari JV, Sugiyama H, McCormick TS and Cooper KD: IL-6 signaling in psoriasis prevents immune suppression by regulatory T cells. J Immunol 183: 3170-3176, 2009.

12. Maggi E, Cosmi L, Liotta F, Romagnani P, Romagnani S and Annunziato F: Thymic regulatory T cells. Autoimmun Rev 4: 579-586, 2005.

13. Wing K, Larsson P, Sandström K, Lundin SB, Suri-Payer E and Rudin A: CD4+ CD25+ FOXP3+ regulatory T cells from human thymus and cord blood suppress antigen-specific $\mathrm{T}$ cell responses. Immunology 115: 516-525, 2005.

14. Wu CT, Chang YH, Lin WY, Chen WC and Chen MF: TGF beta1 expression correlates with survival and tumor aggressiveness of prostate cancer. Ann Surg Oncol 22 (Suppl 3): S1587-S1593, 2015.

15. Vacchelli E, Semeraro M, Enot DP, Chaba K, Poirier Colame V, Dartigues P, Perier A, Villa I, Rusakiewicz S, Gronnier C, et al: Negative prognostic impact of regulatory $\mathrm{T}$ cell infiltration in surgically resected esophageal cancer post-radiochemotherapy. Oncotarget 6: 20840-20850, 2015.

16. Couper KN, Blount DG, de Souza JB, Suffia I, Belkaid Y and Riley EM: Incomplete depletion and rapid regeneration of Foxp3+ regulatory $\mathrm{T}$ cells following anti-CD25 treatment in malaria-infected mice. J Immunol 178: 4136-4146, 2007.

17. Lesokhin AM, Hohl TM, Kitano S, Cortez C, Hirschhorn-Cymerman D, Avogadri F, Rizzuto GA, Lazarus JJ, Pamer EG, Houghton AN, et al: Monocytic CCR2(+) myeloid-derived suppressor cells promote immune escape by limiting activated CD8 T-cell infiltration into the tumor microenvironment. Cancer Res 72: 876-886, 2012.

18. Kersten K, Salvagno C and de Visser KE: Exploiting the immunomodulatory properties of chemotherapeutic drugs to improve the success of cancer immunotherapy. Front Immunol 6: 516, 2015.

19. Zhang H, Li ZL, Ye SB, Ouyang LY, Chen YS, He J, Huang HQ, Zeng YX, Zhang XS and Li J: Myeloid-derived suppressor cells inhibit $T$ cell proliferation in human extranodal NK/T cell lymphoma: A novel prognostic indicator. Cancer Immunol Immunother 64: 1587-1599, 2015.
20. Ding Y, Shen J, Zhang G, Chen X, Wu JM and Chen W: CD40 controls CXCR5-induced recruitment of myeloid-derived suppressor cells to gastric cancer. Oncotarget 6: 38901-38911, 2015.

21. Kitamura T, Qian BZ, Soong D, Cassetta L, Noy R, Sugano G, Kato Y, Li J and Pollard JW: CCL2-induced chemokine cascade promotes breast cancer metastasis by enhancing retention of metastasis-associated macrophages. J Exp Med 212: 1043-1059, 2015.

22. Hefetz-Sela S, Stein I and Pikarsky E: Restoring inflammatory balance as a potential preventive strategy for inflammation induced cancer. Oncoimmunology 4: e1039764, 2015.

23. Rodriguez JA, Huerta-Yepez S, Law IK, Baay-Guzman GJ, Tirado-Rodriguez B, Hoffman JM, Iliopoulos D, Hommes DW, Verspaget HW, Chang L, et al: Diminished expression of CRHR2 in human colon cancer promotes tumor growth and EMT via persistent IL-6/Stat3 signaling. Cell Mol Gastroenterol Hepatol 1: 610-630, 2015.

24. Wolchok JD, Kluger H, Callahan MK, Postow MA, Rizvi NA, Lesokhin AM, Segal NH, Ariyan CE, Gordon RA, Reed K, et al: Nivolumab plus ipilimumab in advanced melanoma. N Engl J Med 369: 122-133, 2013.

25. Rizvi NA, Mazières J, Planchard D, Stinchcombe TE, Dy GK, Antonia SJ, Horn L, Lena H, Minenza E, Mennecier B, et al: Activity and safety of nivolumab, an anti-PD-1 immune checkpoint inhibitor, for patients with advanced, refractory squamous non-small-cell lung cancer (CheckMate 063): A phase 2, single-arm trial. Lancet Oncol 16: 257-265, 2015.

26. Robert C, Long GV, Brady B, Dutriaux C, Maio M, Mortier L, Hassel JC, Rutkowski P, McNeil C, Kalinka-Warzocha E, et al: Nivolumab in previously untreated melanoma without BRAF mutation. N Engl J Med 372: 320-330, 2015.

27. Kazandjian D, Khozin S, Blumenthal G, Zhang L, Tang S, Libeg M, Kluetz P, Sridhara R, Keegan P and Pazdur R: Benefit-risk summary of nivolumab for patients with metastatic squamous cell lung cancer after platinum-based chemotherapy: A report from the US Food and Drug Administration. JAMA Oncol 2: 118-122, 2016.

28. Mountzios G, Linardou $\mathrm{H}$ and Kosmidis P: Immunotherapy in non-small cell lung cancer: The clinical impact of immune response and targeting. Ann Transl Med 4: 268, 2016.

29. Kong YC and Flynn JC: Opportunistic autoimmune disorders potentiated by immune-checkpoint inhibitors Anti-CTLA-4 and Anti-PD-1. Front Immunol 5: 206, 2014.

30. Nishino M, Sholl LM, Hodi FS, Hatabu H and Ramaiya NH: Anti-PD-1-related pneumonitis during cancer immunotherapy. N Engl J Med 373: 288-290, 2015.

31. Finkelman FD, Lees A and Morris SC: Antigen presentation by B lymphocytes to CD4+ T lymphocytes in vivo: Importance for B lymphocyte and T lymphocyte activation. Semin Immunol 4: 247-255, 1992.

32. Brentville VA, Metheringham RL, Gunn B, Symonds P, Daniels I, Gijon M, Cook K, Xue W and Durrant LG: Citrullinated vimentin presented on MHC-II in tumor cells is a target for CD4+ T cell-mediated antitumor immunity. Cancer Res 76: 548-560, 2016.

33. Zhong J, Chen Y, Liao X, Li J, Wang H, Wu C, Zou X, Yang G, Shi J, Luo L, et al: Testis expressed 19 is a novel cancer-testis antigen expressed in bladder cancer. Tumour Biol 37: 7757-7765, 2016.

34. Wang L, Lin J, Zhou Z, Huo R, Shen B, Sun Y and Li N: Up-regulation of Th17 cells may underlie inhibition of Treg development caused by immunization with activated syngeneic T cells. PLoS One 6: e27289, 2011.

35. Andtbacka RH, Kaufman HL, Collichio F, Amatruda T, Senzer N, Chesney J, Delman KA, Spitler LE, Puzanov I, Agarwala SS, et al: Talimogene laherparepvec improves durable response rate in patients with advanced melanoma. J Clin Oncol 33: 2780-2788, 2015.

36. Janke M, Peeters B, de Leeuw O, Moorman R, Arnold A, Fournier P and Schirrmacher V: Recombinant newcastle disease virus (NDV) with inserted gene coding for GM-CSF as a new vector for cancer immunogene therapy. Gene Ther 14: 1639-1649, 2007. 